\title{
An Intelligent Algorithm for Negative Sequence Directional Element of DFIG during Ferroresonance in Smart Grid
}

\author{
Salman Rezaei ${ }^{1}$ (D) \\ Received: 10 February 2021 / Accepted: 5 November 2021 / Published online: 13 December 2021 \\ (c) The Author(s), under exclusive licence to Springer Nature Singapore Pte Ltd. 2021
}

\begin{abstract}
Smart grid comprises utility system and Distributed renewable power Generations such as wind and solar energy. The smart grid system represents bidirectional flowing of energy and communication facilities among utility system, Distributed Generations (DG) and consumers. Smart grid including wind farm is seriously exposed to high magnitudes of nonlinearities like ferroresonance. It causes mal operation of protective relays in wind farm. This paper investigates impact of ferroresonance in utility system on operation of DFIG (Doubly-Fed Induction Generator) and Negative Sequence Directional Element (NSDE) in Wind Park by means of PSCAD/EMTDC software. As smart grid insists on a self-healing protection, an intelligent algorithm based on wavelet transform, neural network and ferroresonance analysis in time domain is proposed for NSDE to discriminate ferroresonance. The algorithm appropriately conforms to smart grid protection strategy. It discriminates ferroresonance from other nonlinear abnormalities and is able to distinguish different types of ferroresonance. To accord with smart grid requirements, the algorithm is designed to forecast occurrence of ferroresonance in the grid.
\end{abstract}

Keywords Intelligent algorithm $\cdot$ Negative sequence directional element $\cdot$ Ferroresonance $\cdot$ Smart grid $\cdot$ Distributed generations

\section{Introduction}

Smart grid mostly insists on self-healing attribute, which is capable of increasing reliability, preserves voltage magnitude, and improves asset management. It employs dynamic optimization techniques that utilize real time measurements in the network [1]. Self-healing protection systems take advantage of equipment that recovers the power automatically in case of any abnormality or a fault in the system. This decreases the number of consumers affected by power outage. The beneficial aspects of self-healing protection in smart grid are stated in [2].

Renewable energy generations such as wind turbines are particularly vulnerable to ferroresonance. Ref. [3] analyzes ferroresonance in wind turbine in islanding mode. Influence of ferroresonance in islanding on different kinds of generators in wind farm is stated in [4]. Ferroresonance in effect of

Salman Rezaei

rezaiesalman@gmail.com

1 Technical Office, Kerman Power Generation Management Co, Kerman, Iran existing capacitance, which is utilized for VAR compensation in Wind Park, is expressed in [5].

In order to discriminate different kinds of faults in wind farm and protection of DFIG, variety of methods and algorithms have been presented in technical literature. Ref. [6] utilizes positive and negative measured values to discriminate faults inside or outside of wind farm. Directional overcurrent element is employed in [7] to protect generator in wind farm. Ref. [8] represents a state of the art overcurrent protection based on wide area measurement in smart grid. Adaptive algorithm for microprocessor based distance relays applicable in smart grid is proposed in [9].

In addition to that, adaptive protection in smart grid gains a lot of attention in technical literature. Ref. [10] proposes the mitigation of distributed generation impact on directional overcurrent relay coordination by employing adaptive protection scheme using differential evolution algorithm, which causes improving overall sensitivity of relays. Ref. [11] assesses the requirements for selectivity and sensitivity of an overcurrent relay protection against phase-to-phase faults at connection of renewable energy sources.

Wavelet transform is used widely in area of protection in smart grid. Ref. [12] investigates a novel hybrid 
technique consisting of wavelet packet transform (WPT), in addition, direct and quadrature (dq) axis components in order to find out and evaluate the best signature of the internal faults. A new technique for fault classification and detection in the transmission lines of micro-grids using a Discrete Wavelet Transform (DWT) and a Back-Propagation Neural Network (BPNN) is proposed in [13]. Ref. [14] analyzes the effectiveness of a wavelet based protection strategy under dynamic and transient conditions within a micro grid.

Several papers represent new methods of electrical protection in micro grids including wind farm. Ref. [14] presents the simulation and modeling process of an overcurrent protection scheme with pre-established configurations and centralized control of such configurations. A novel transient current similarity-based protection scheme, which uses the fundamental frequency and non-fundamental frequency characteristics along with transient current, is proposed in [15]. Moreover, a stand-alone Wind farm relay (WFR) of collector feeders in wind farm based on directional angle algorithm and positive sequence current is introduced in [16].

Analysis of ferroresonance in renewable energy resources including wind farm is carried out in literature. Ref. [17] studies the transient and sustained ferroresonance phenomenon in wind farms connected to a power distribution system. Moreover, an analysis about the occurrence and the severe effects of ferroresonance during islanding mode of micro grid is presented in [18].

Ferroresonance brings about increment of voltage and current in the grid. It consequently leads in thermal impact and catastrophic damage to the insulation. Among this, protective relays are also affected due to increasing electrical quantities [19]. It effectively causes mal operation or restriction of operation of the protective function when the relay is required to operate to disconnect vulnerable or faulty areas. Hence, the current-based relays proposed in [6-8] protects wind turbine against internal and external faults. However, operation of the relays is not reliable in nonlinear conditions like ferroresonance.

NSDE is utilized to discriminate unsymmetrical faults inside or outside of Wind Park. The relay must be able to determine behavior of protective function whether it picks up or not during ferroresonance. A conventional relay with a setting quantity and even input signal for ferroresonance detection is not able to make a decision on behavior of the relay, where operation or restriction of operation is necessarily required during ferroresonance. Hence, an adaptive algorithm is essential to make a decision on behavior of the function in such conditions.

The above-mentioned references regarding intelligent protection in smart grid and micro grid neither investigate impact of ferroresonance on mal operation of NSDE nor represent a solution to overcome the problem in smart grid or micro grid.
Adaptation of NSDE in ferroresonance has been firstly sketched out in [20]. Occurrence of ferroresonance in traditional network can be discriminated by the algorithm and relay setting quantities are modified according to constant coefficients determined by user. However, the algorithm could not be sufficiently adaptable to decide on behavior of the protective relay based on the results of computational analysis in the network to improve stability and availability of the smart grid in abnormal conditions.

Conference version of this paper [21] came up with the idea of enhancing investigations respecting more adaptation of NSDE and extended application of the algorithm in smart grid. Application of wavelet to classify faults is expressed in [12-14]. While the algorithm presented in this work exclusively utilizes DWT and neural network to distinguish ferroresonance from other nonlinearities in the network. Hence, the algorithm is well designed to address specific requirements in terms of receiving modification factors from protection strategy and consequently automatic modification of the relay as well as provided in a closed-loop system.

This paper has been significantly developed compared to its conference version. Theoretical aspects of NSDE, ferroresonance detection on the basis of wavelet and neural network, in addition, time domain analysis to distinguish ferroresonance of different types is added to this version. Furthermore, the structure of protection strategy and issue of protection strategy logic are specifically explained in more details.

Thereafter, influence of ferroresonance in effect of line and plant outage is analyzed on operation of DFIG by PSCAD/EMTDC software. Furthermore, behavior of NSDE is assessed during ferroresonance in wind farm. Then, the proposed algorithm is implemented by the software to recognize ferroresonance of different types. Modification command is sent to the algorithm from protection strategy to take a suitable decision on behavior of the relay during ferroresonance. It modifies relay setting quantities to restrain mal operation or leads in mandatory operation of the relay based on protection strategy in smart grid.

Smart grid stands on need of a predictive protection, therefore; the algorithm is utilized to forecast ferroresonance of different types based on variation of voltage in the grid. Finally, the algorithm is examined in ferroresonance condition to confirm the correct operation.

\section{Theoretical Approaches}

\section{Negative Sequence Directional Element}

Generally, recognition of unbalance fault direction requires a separate directional element. It is proven that in order to discriminate an unbalance fault, positive and negative sequence quantities are required. In fact, directional elements are 
misguided in effect of positive sequence quantities. Hence, unbalance faults can only be distinguished by negative sequence quantities as input measurements to unbalance fault directional relays. Traditional negative sequence component and corresponding operating torque (T32Q) are calculated by the following formulas [22-26].

$3 V_{2}(t)=V_{A}(t)+a^{2} V_{B}(t)+a V_{c}(t)$

$3 I_{2}(t)=I_{A}(t)+a^{2} I_{B}(t)+a I_{c}(t)$

$T 32 Q=\left|3 V_{2}\right| \cdot\left|3 I_{2}\right| \cos \left(\angle-3 V_{2}-\left(\angle 3 I_{2}+\angle M T A\right)\right.$

where: $a=1\left\llcorner 120^{\circ}\right.$

MTA $=$ Maximum Torque Angle

It can be inferred that positive operating torque indicates forward direction, reciprocally; negative operating torque is the evidence of a reverse direction. An improved method to determine unbalance fault direction is attained by consideration of negative sequence impedance directional element, which utilizes negative sequence measuring components. The NSDE measures the value and phase of $I_{2}, V_{2}$ and value of $I_{1}$. Setting quantities like; forward and reverse overcurrent, $Z_{2}$ forward and $Z_{2}$ reverse threshold values, restrain factor of $I_{1}$ and impedance line angle are determined. Then the relay calculates negative sequence impedance $\left(\mathrm{Z}_{2}\right)$ by the following formula.

$Z_{2}=\frac{V_{2}}{I_{2}}=\frac{3 V_{2}}{3 I_{2}}$

The NSDE operates based on the principle that in case of measuring the negative value of $Z_{2}$ forward fault is detected, whereas, a positive value of $Z_{2}$ indicates reverse fault. The relay is activated as soon as the ratio of negative sequence current to positive sequence current $\left(\mathrm{I}_{2} / \mathrm{I}_{1}\right)$ exceeds the threshold value. Furthermore, $3 \mathrm{I}_{2}$ must be greater than forward and reverse setting quantities for detection of both forward and reverse faults respectively. According to setting quantities of the relay, $Z_{2}$ forward and $Z_{2}$ reverse are defined then the criteria is that if $Z_{2}<Z_{2}$ forward, then the fault is forward and if $Z_{2}>Z_{2}$ reverse, then the fault is reverse. In order to avoid any overlap, the forward threshold must be less than the reverse threshold [27].

\section{Principle of ferroresonance detection based on wavelet and neural network}

Wavelet and neural network are utilized in power system to distinguish ferroresonance from other nonlinear abnormalities. The method explained in [28] works based on wavelet and neural network. It discriminates ferroresonance from other transient abnormalities like; transformer energizing current including second harmonic, in addition, chopping and strike current due to reactor and capacitance switching. Discrete Wavelet Transform (DWT) analyses the signal at different frequency bands with different resolutions by decomposition of the signal into a course of approximation and detail information.

Decomposition of the signal is obtained by successive high pass and low pass filtering of the corresponding signal in time domain. The original signal $x[n]$ is passed throughout a half band high pass filter $\mathrm{g}[\mathrm{n}]$ and a low pass filter $\mathrm{h}[\mathrm{n}]$. After the filtering process, half of the samples are removed based on the Nyquist's principle, since the signal now has a highest frequency of a half value of original signal. The signal can therefore be subsampled by 2 directly by truncating every other sample. This establishes one level of decomposition expressed by the following formulas.

$y h i g h[k]=\sum_{n} x[n] . g[2 k-n]$

$y l o w[k]=\sum_{n} x[n] \cdot h[2 k-n]$

The above-mentioned process (known as the sub band coding) is able to repeat up to desired level of decomposition. Variety of mother wavelet families like; Harr, Daubechies, Symlets and Coeiflets are presented and abbreviated by a surname ('har', 'db', 'sym' and 'coif') and a number, which follows the surname to represent the order. More details respecting wavelet transform is stated in [29].

Neural network comprises interconnected adaptive simple elements (neurons), which are responsible to execute parallel calculation for data processing. The characteristic of self-organization of networks is an important matter in neural network systems. Such networks can recognize the organization and connectivity, which can be presented at the input and will be able to respond to the other inputs based on that organization. Neural network distinguishes the pattern, and fits and clusters the data. Structure of neural network comprises input layer, layer of neurons (hidden layer), and output layer. Input data are used for accomplishment of training, validation and testing procedure. The weighs (w) are changed in order to match input data based on target data to attain desired output by selecting an appropriate training algorithm during training course [30].

As was discussed in advance, the method is improved to discriminate ferroresonance from other nonlinear abnormalities in smart grid. One particularly prominent example of this issue can be effect of electronic devices in renewable energy plants (like inverter and converter) on power quality of the grid. This can be accomplished by utilizing wavelet and neural network in the system. A great deal of samples, which are designated to definite nonlinear conditions are considered to train neural network. Application of wavelet and neural network is shown in the next section. 


\section{Ferroresonance detection algorithm based on time domain analysis}

Determination of ferroresonance of different types is performed in time domain. It is accomplished after discrimination of ferroresonance from other abnormal conditions in the network. Ref. [31-34] represents analysis of ferroresonance in time and frequency domain.

Ref. [35] and [36] proposes a different method based on time domain analysis described as follows. The criteria to determine ferroresonance of different types can be attained by calculation of frequency difference $(\Delta f), d \Delta f / d t$, and Total Harmonic Distortion (THD) of current or voltage waveform. The criteria to determine ferroresonance of different types based on the $\Delta \mathrm{f}$ and THD with typical values are illustrated in Table 1.

Generally, electronic devices increase the value of THD. In the same manner, the THD increases significantly during ferroresonance in the grid. In this study, in order to appropriate recognition of ferroresonance in renewable energy plants including inverter and converter one additional criteria based on the quantity of dTHD/dt is considered for detection of ferroresonance. Application of such criteria to design an adaptive algorithm for NSDE is described in the next section.

\section{Methods}

In this section, an adaptive algorithm is proposed for NSDE during ferroresonance in smart grid.

\section{Introduction}

Smart grid including renewable power generations, which substantially integrates adaptive devices to establish selfhealing characteristic, hence, a self-healing protection is also required in the grid. Therefore, an adaptive algorithm is essential to be compatible with smart grid requirements to distinguish ferroresonance from other similar phenomena. To accomplish this, theory of an adaptive algorithm is proposed for NSDE.

Ferroresonant states must fairly be distinguished from other phenomena to become protection strategy capable of relating measured quantities to specific abnormal conditions in the network. Consequently, the protection strategy will be able to make a suitable decision to determine behavior of the relay in each condition.

As stated in the above-mentioned references respecting ferroresonance, it brings about increasing voltage and current in the network. Like state of the art protective relays, NSDE utilizes fundamental harmonic to filter out high frequency interferences. The relay may conduct mal operation in effect of increasing the value of current whereas, no fault occurs in the system. On the other hand, the relay probably does not operate due to decreasing fundamental value in severe ferroresonant states, whereas, true rms value of current increases exponentially and damages the equipment so that, the relay has to operate properly to interrupt the circuit.

Protection strategy is generally responsible to control behavior of protective relays in different conditions based on power stability and availability of power generations in smart grid. It is obtained by results of electrical studies in the network. Hence, protection strategy urges the relay whether to operate or restrict the operation in case of ferroresonance to stabilize the grid. In order to adapt the relay to smart grid requirements, NSDE is equipped with an intelligent algorithm. Structure of the algorithm and protection strategy is explained in this section.

\section{Structure of the algorithm}

Structure of the adaptive algorithm is shown in Fig. 1. It adapts behavior of the NSDE during ferroresonance to protection strategy requirements of smart grid. Wavelet and neural network are utilized to discriminate ferroresonance from other abnormalities due to operations like; transformer energizing, load and capacitance switching. Along with recognition of what mentioned above, the method is enhanced in the proposed algorithm to discriminate ferroresonance from influence of electronic devices in renewable energy plants. The current signal is decomposed into a course of approximation and detail information up to six levels by daubechies $(\mathrm{db})$ mother wavelet, which is more practical in such applications.

According to above-mentioned abnormalities, five classes of detections are obtained by clustering the input signals based on patterns provided for each class. Pattern (target) signals are entered into the algorithm in order to train neural
Table 1 Criteria to determine ferroresonance mode

\begin{tabular}{llllll}
\hline Ferroresonance type & $\Delta \mathrm{f}(\mathrm{Hz})$ & $\frac{\mathrm{d} \Delta \mathrm{f}}{\mathrm{dt}}$ & T.H.D $(\%)$ & $\frac{\mathrm{dTHD}}{\mathrm{dt}}$ & Harmonic spectrum \\
\hline Fundamental & Zero & Zero & $50>$ & $5>$ & Discrete \\
Harmonic & Constant & Zero & $50>$ & $10>$ & Discrete \\
Quasi-periodic & Variable & Not zero & $100>$ & $35>$ & Discrete \\
Chaotic & Variable & Not zero & $100>$ & $35>$ & Continuous \\
\hline
\end{tabular}


Fig. 1 Adaptive algorithm proposed for NSDE

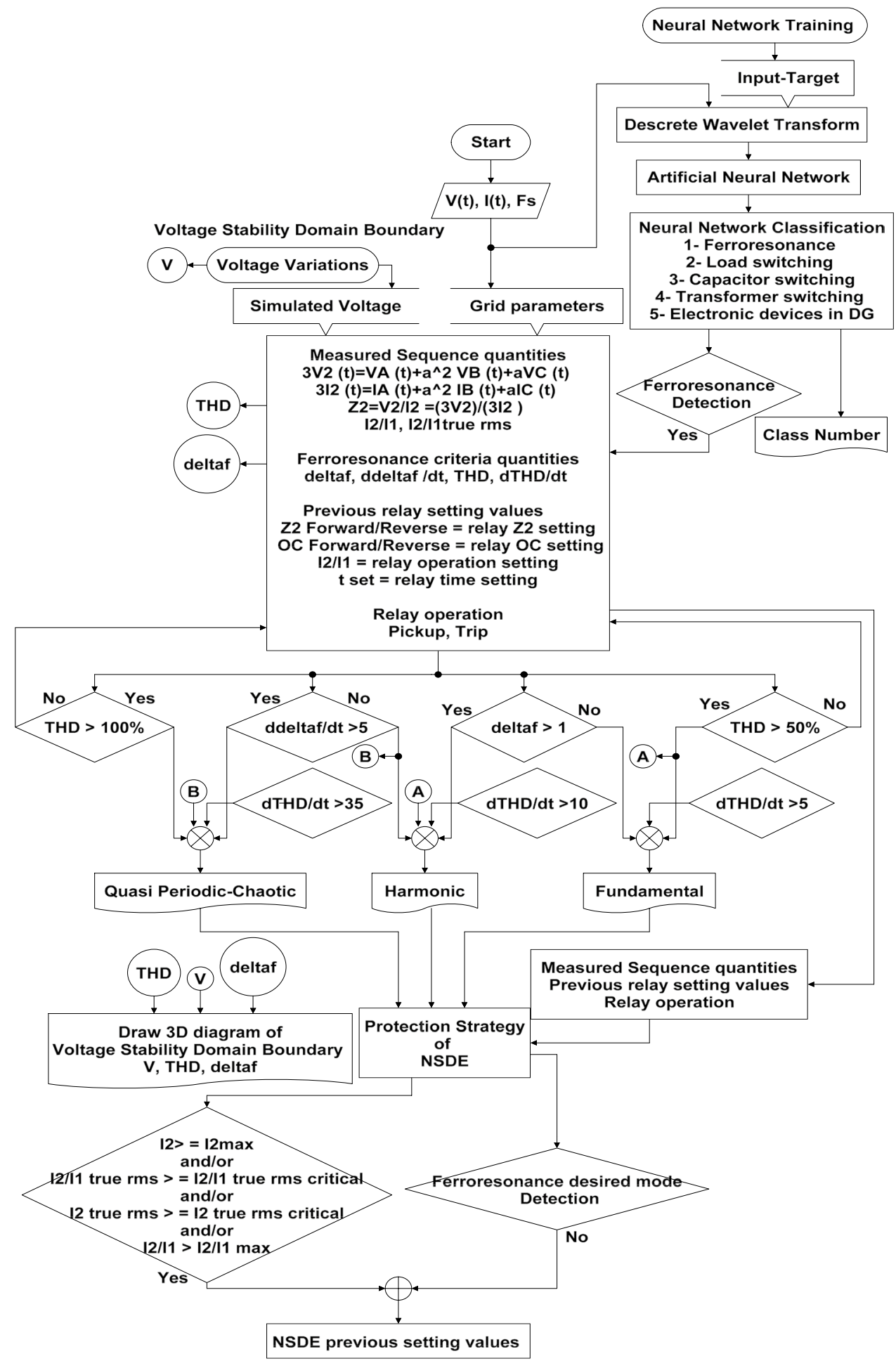

network in commissioning term. It is evident that including more patterns to the algorithm results in more successful training of the neural network in this term. The algorithm is designed to discriminate ferroresonance from other transient or steady state abnormalities. Furthermore, ferroresonance of different types is determined and utilized by protection strategy to decide the ferroresonance type, in which relay setting modification must be implemented [39, 40].

Firstly, the algorithm measures fundamental values of positive and negative sequence of voltage and current like; $\mathrm{V}_{2}, \mathrm{I}_{2}, \mathrm{I}_{1}$, and true rms values of $\mathrm{I}_{2}$ and $\mathrm{I}_{1}$. Then the quantities like $Z_{2}, I_{2} / I_{1}$ and $I_{2} / I_{1}$ true rms are calculated. In addition 
to that, previous relay setting quantities are also considered by the algorithm. Such information is used by protection strategy of NSDE to take a suitable decision on behavior of the relay during ferroresonance. Previous relay setting quantities are achieved when specified type of ferroresonance cannot be detected or one of the condition based on threshold values like; $\mathrm{I}_{2} \mathrm{max}, \mathrm{I}_{2} / \mathrm{I}_{1}$ true rms critical, $\mathrm{I}_{2}$ true rms critical and $\mathrm{I}_{2} / \mathrm{I}_{1}$ max are fulfilled.

Furthermore, stability domain boundary of voltage is determined by the algorithm based on the THD and $\Delta \mathrm{f}$. This characteristic is organized so the values of THD and $\Delta \mathrm{f}$ are changed versus variation of simulated voltage. Consequently, the ferroresonance of different types are determined in each value of simulated voltage. The algorithm performs the process in normal status to warn the probable ferroresonance condition in the network configuration. The proposed algorithm comprises a logical circuit, which acts in case of any kind of misshaped signal whether obtained from a disturbance in a bulk power system or provided in laboratory scale.

The algorithm proposed in this section is compared to the similar state of the art relays and algorithms against faults in wind farms stated in the literature.

The algorithm expressed in [15] uses the fundamental and non-fundamental frequency characteristics along with transient currents during the faults to identify magnetizing currents and internal transformer faults in a short period in wind farm. It also recognizes low-order components that may affect the second-harmonic restraint algorithm of the transformer differential protection. Similarly, the proposed algorithm in this section uses the fundamental and non-fundamental frequency characteristics of the current or voltage. However, it recognizes occurrence of nonlinearities especially ferroresonance in wind farm and increases reliability of NSDE in wind farm.

Moreover, the method stated in [16], recognizes internal and external faults in wind farm based on directional angle algorithm and positive sequence current, while the proposed algorithm in this section recognizes internal and external faults in wind farm intellectually even if ferroresonance occurs in the system. It modifies NSDE setting quantities to prevent mal operation of the relay during ferroresonance.

\section{Structure of the protection strategy}

As was discussed in advance, behavior of the relay is determined by protection strategy based on security and dependability of protection and consideration of stability and availability of the grid at the time of ferroresonance. In this section, structure of the protection strategy based on behavior of the relay in ferroresonance is described in two conditions as follows.

\section{Prevent mal operation of the relay during ferroresonance}

As will be illustrated subsequently, ferroresonance leads in increasing the quantity of negative sequence of the current compared to positive sequence. It causes successive activation of pickup signal of the relay at both directions. In some cases, protection strategy urges to restrict trip operation of the relay in ferroresonance condition. Figure 2 illustrates structure of protection strategy algorithm to prevent mal operation of the relay during ferroresonance.

Protection Strategy Logic (PSL) is responsible to specify setting quantities of the algorithm according to results of electrical studies implemented in the network, practical experiences and technical compromises, which determine stability, and necessity of availability of the grid in each condition [41]. By means of the studies, it is also practicable to forecast consequences of activation or restriction of the relay output in different phenomena (ferroresonance) to take a correct decision in such conditions. It is noted that explanation of PSL in detail is out of scope of this study.

Detection of ferroresonance along with pickup operation of the relay causes activation of the algorithm to modify setting quantities. The algorithm is responsible to determine the ferroresonance mode, in which setting quantities of the relay have to be modified. It is notified that PSL may decide to modify setting quantities especially in severe ferroresonant states (like quasi-periodic or chaotic). It is evident that the relay picks up when $\mathrm{I}_{2} / \mathrm{I}_{1}$ and $3 \mathrm{I}_{2}$ are greater than setting values. Therefore, both setting quantities must be modified to prevent mal operation of the relay.

In case of increasing the measured values of $\mathrm{I}_{2} / \mathrm{I}_{1}$ and $3 \mathrm{I}_{2}$ higher than the setting quantities and lower than the maximum threshold values the algorithm decides to increase setting quantities to prevent mal operation of the relay during ferroresonance. The measured quantities of $\mathrm{I}_{2} /$ $\mathrm{I}_{1}$ and $3 \mathrm{I}_{2}$ are multiplied by PIF (Pickup Increasing Factor) and SF (Security Factor), which are determined by PSL. Therefore, OC (Over Current) reverse or forward setting quantities are increased according to direction of operation. It is notified that maximum threshold quantities are established by PSL. In case of increasing the above setting quantities greater than defined values (specified by PSL) PSL decides to increase trip time. Then the algorithm multiplies previous time setting by TIF (Time Increasing Factor), which is also specified by PSL. In some cases, the PSL may decide to block trip operation to prevent mal operation of the relay during ferroresonance. 
Fig. 2 Protection strategy to prevent mal operation of NSDE

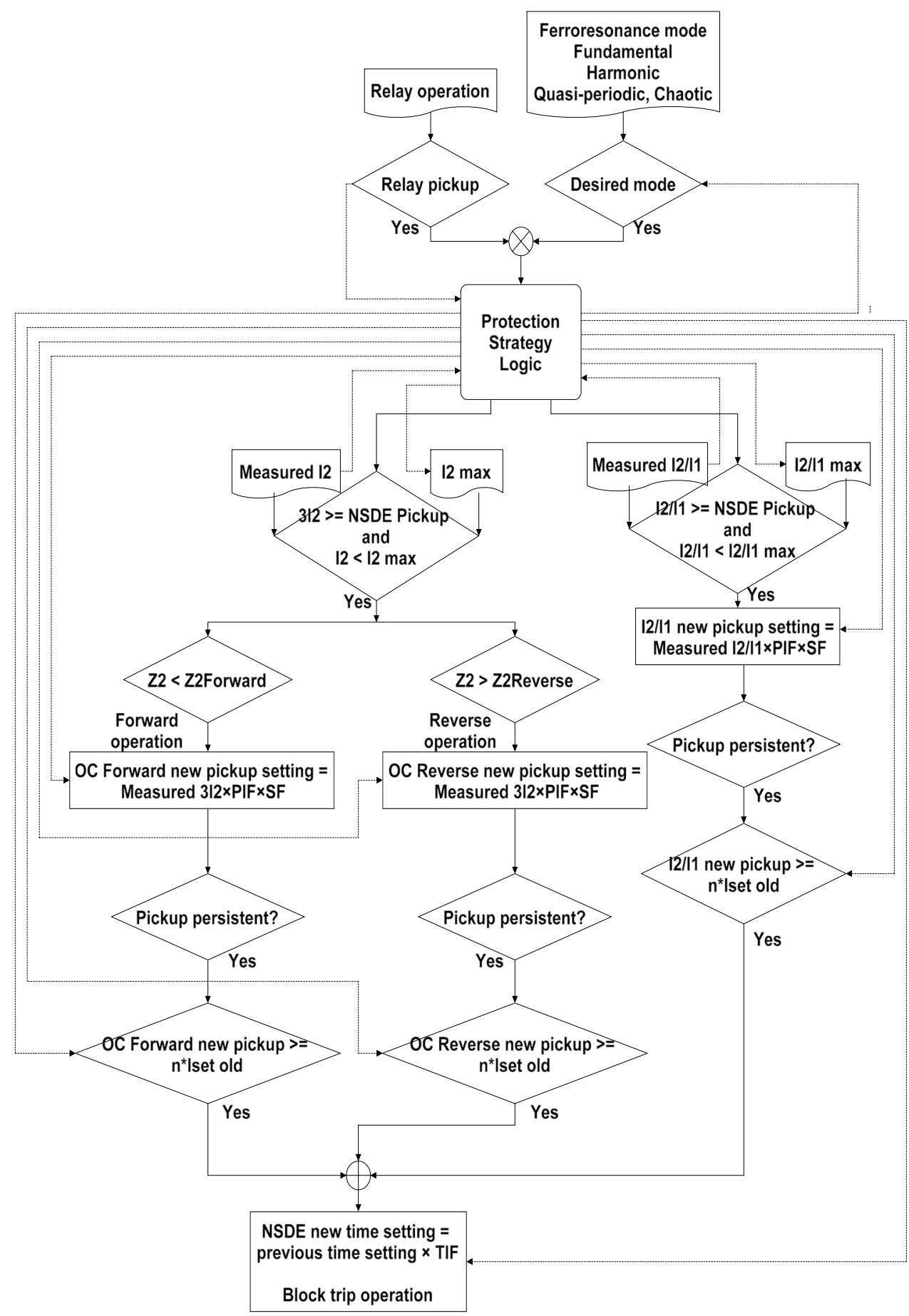

\section{Prevent restriction of operation of the relay during ferroresonance}

As was discussed in advance, it is highly likely that the fundamental harmonic decreases in severe ferroresonant states like quasi-periodic or chaotic mode. Consequently, the relay may not pick up properly in such condition. Whereas, due to exponential increment of the true rms value of current, trip output of the relay must be activated according to PSL requirements. Figure 3 illustrates structure of protection strategy algorithm, which prevents restriction of the relay in ferroresonance.

As soon as exceeding true rms values of $\mathrm{I}_{2} / \mathrm{I}_{1}$ or $3 \mathrm{I}_{2}$ from specified maximum threshold values specified by PSL in presence of ferroresonance, the algorithm decides to decrease pickup setting of the relay by multiplying the 
measured values of $\mathrm{I}_{2} / \mathrm{I}_{1}$ and $3 \mathrm{I}_{2}$ by PDF (Pickup Decreasing Factor) and SF (Security Factor) to operate the relay. Furthermore, the algorithm measures the quantity of $Z_{2}$ true rms to determine modification of forward or reverse direction. PSL considers individual value of PDF for each setting quantity.
As shown by the algorithm, PDF is explicitly an inverse function of true rms values of $\mathrm{I}_{2} / \mathrm{I}_{1}$ or $3 \mathrm{I}_{2}$. Slope of the function is also specified by PSL. If modification of $\mathrm{I}_{2} / \mathrm{I}_{1}$ and $\mathrm{OC}$ reverse or forward setting quantities leads in pickup operation of the relay, PSL decides to specify new trip time setting. Hence, the algorithm multiplies
Fig. 3 Protection strategy to prevent restriction of operation of NSDE

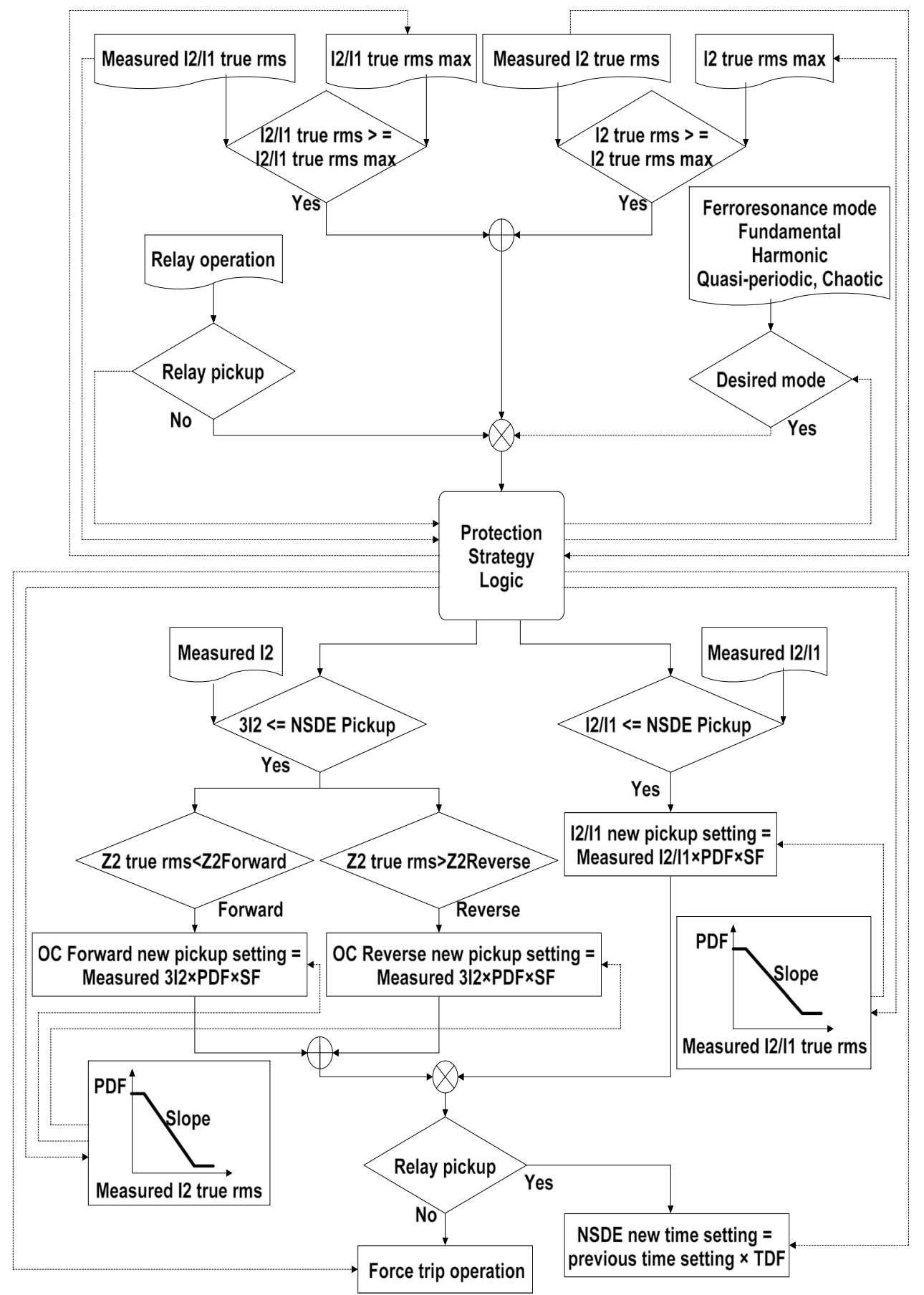


previous time setting by TDF (Time Decreasing Factor) to decrease operating time. If the relay does not pick up by setting modification, then PSL mandatory forces trip operation.

\section{Experimental Studies}

In this section, probability of occurrence of ferroresonance in smart grid is examined and then impact of ferroresonance is analyzed on operation of DFIG and behavior of NSDE.

\section{Introduction}

Renewable power generations such as solar and wind turbines are highly penetrated into smart grid to supply the loads. In this study, wind farm is modelled by a 400 MVA DFIG (Fig. 4). A two-mass Wind turbine is directly coupled with a generator. The DFIG is connected to the HV power network via a $230 / 13.8 \mathrm{kV}$ power transformer. AC/DC converters are connected to rotor and stator of DFIG. It aims the generator to rotate at variable wind speed to generate power at constant voltage and frequency values. The system is examined to analyze the influence of ferroresonance due to line and plant outage in utility system on electrical and mechanical quantities of DFIG in Wind Park. Thereafter, behavior of NSDE is analyzed during ferroresonance.

\section{Impact of ferroresonance on operation of DFIG}

Ref. [37] insists on occurrence of ferroresonance by several different configurations like; power transformer supplied accidentally through one or two phases in effect of breaker phase failure, opening grading capacitance circuit breakers, voltage transformer connected to an ungrounded neutral system, power transformer connected to a series compensated transmission line and so on. In addition, ref [36-38] explain occurrence of ferroresonance due to line and plant outage in the network, which is examined in this study. Generally, line and plant outage in electrical network leads in open end line in the network. It potentially causes overvoltage at the end of the line. In case of existing no-load transformers at the end of the line, transformer is saturated and capacitance line of the system may traverse across nonlinear area of the core characteristic, thus ferroresonance occurs in the system.

There are $230 \mathrm{kV}$ no-load transformers in utility system, to which wind farm is connected. Ferroresonant configuration is already formed by opening designated lines. As shown in Fig. 5, ferroresonance occurs at the time of 0.05 $\mathrm{S}$ from beginning the simulation. Ferroresonance leads in

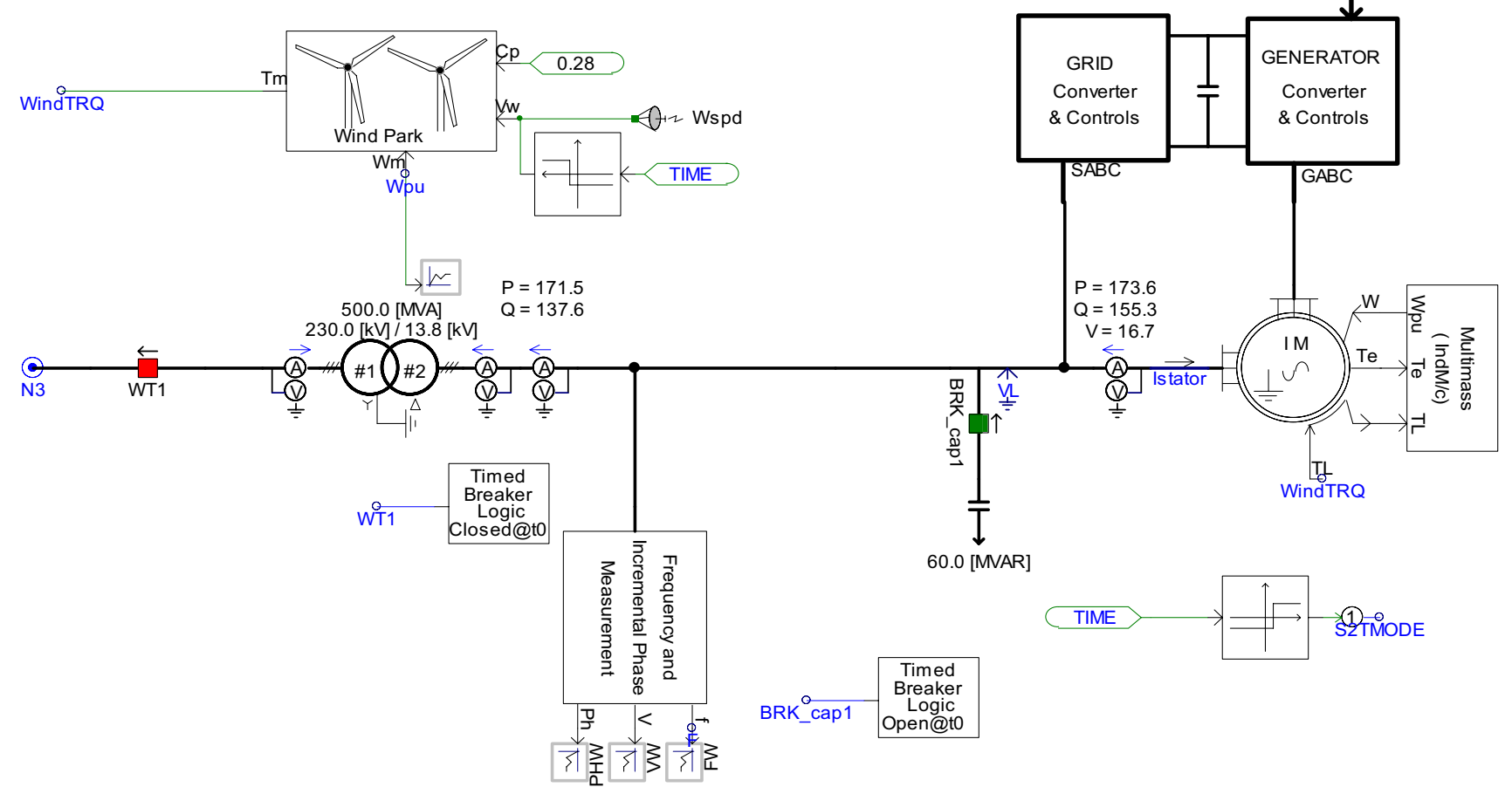

Fig. 4 400MVA wind farm connected to $230 \mathrm{kV}$ network 


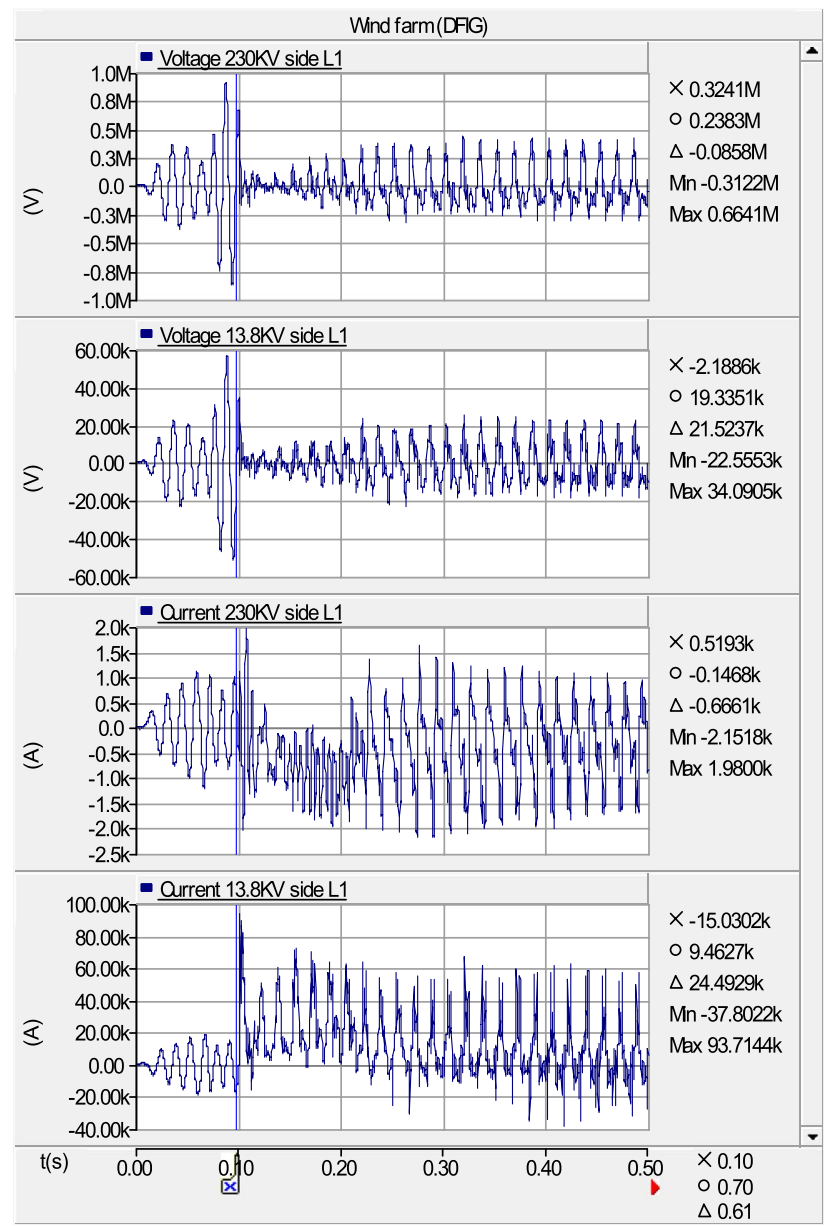

Fig. 5 Electrical quantities of wind farm in ferroresonance

increasing and misshaping voltage and current waveforms in the grid (230 kV side) and in wind farm ( $13.8 \mathrm{kV}$ side).

Ferroresonance in wind farm also leads in increasing and oscillating mechanical quantities of DFIG. As shown in Fig. 6, multi-mass shaft model is activated at the time of $0.4 \mathrm{~S}$. it causes deviation of mechanical quantities due to ferroresonance. At this time, mechanical quantities of wind turbine increase markedly. Mechanical torque on shaft rises up to $4 \mathrm{pu}$ and mechanical position of turbine is deviated up to $1^{\circ}$ with respect to generator. As it is evident from the graph provided, mechanical quantities like, speed, torque on shaft and mechanical position between masses oscillate between positive and negative values. Oscillation of quantities with high frequency at both directions leads in catastrophic mechanical damage and shaft fatigue, which has cumulative effect.

\section{Impact of ferroresonance on behavior of NSDE}

Ferroresonance in effect of line and plant outage is apparently a symmetrical phenomenon; nevertheless,

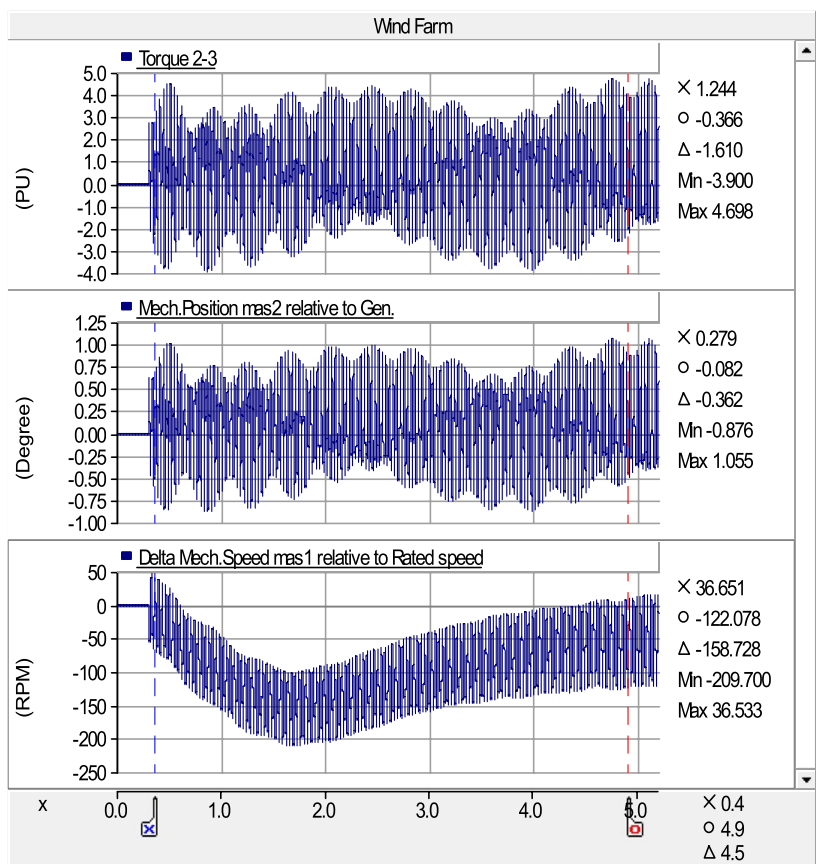

Fig. 6 Mechanical quantities of wind farm in ferroresonance

decomposition of the current into symmetrical components shows a considerable amount of negative sequence component due to deviation of waveforms in ferroresonance. Figure 7 shows mal operation of NSDE during ferroresonance.

As illustrated by the graph, transient ferroresonant state brings about increasing the quantities of $\mathrm{I}_{2} / \mathrm{I}_{1}$ and $\mathrm{I}_{2}$ at the instance of line and plant outage. It causes consecutive pickup and drop off operation of the relay during ferroresonance. In normal condition, the quantities of $\mathrm{I}_{2} / \mathrm{I}_{1}$ and $3 \mathrm{I}_{2}$ are 0.04 and $0.06 \mathrm{~A}_{\mathrm{sec}}$ (ampere in secondary side of instrument transformer) respectively; however, these values respectively increase up to 0.17 and $0.185 \mathrm{~A}_{\mathrm{sec}}$ in ferroresonance. In such conditions, the relay trip output is activated due to persistence of pickup value more than trip time. Although transformer is unloaded in this situation, mis-operation of the relay causes transformer becomes unavailable for further loading condition. It is also interesting to note that, ferroresonance may occur in light load condition; hence, transformer outage leads in de-energization of light loads.

\section{Sensitivity analysis of the ferroresonance classification}

As was discussed in advance, the criteria to determine ferroresonance of different types is obtained by the parameters like; frequency difference $(\Delta \mathrm{f}), \mathrm{d} \Delta \mathrm{f} / \mathrm{dt}$, Total Harmonic Distortion (THD) and dTHD/dt of current or voltage waveform. Ferroresonance classification algorithm uses variety of analogue components like, measuring devices, and analysis 


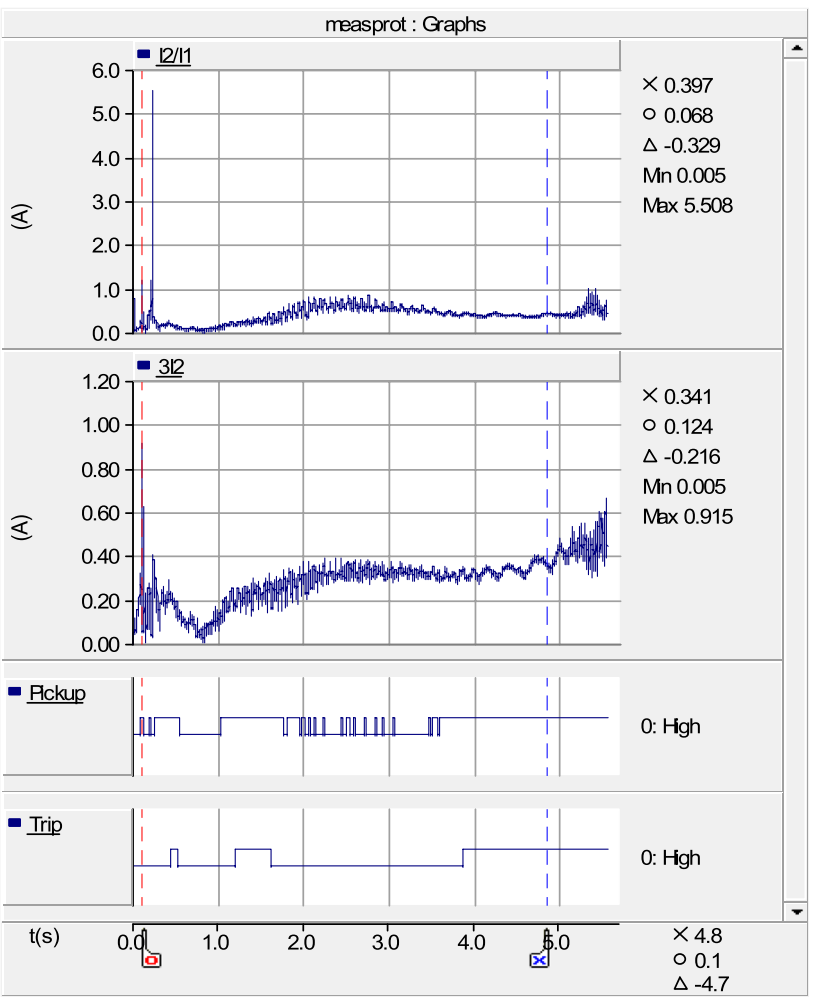

Fig. 7 Mal operation of NSDE in ferroresonance

tools, along with digital components like comparator, and logic gates.

Sensitivity of operation of ferroresonance classification algorithm depends on accuracy and precision of measuring and calculating components like; instrument transformers, Fast Fourier Transform (FFT), and derivative tools. In order to perform sensitivity analysis of the algorithm, quantities like $\Delta \mathrm{f}$ and THD measured by the algorithm are compared to reference quantities obtained by the software database to calculate the error values of the algorithm. Then, the accuracy of derivative components, which calculate $\mathrm{d} \Delta \mathrm{f} / \mathrm{dt}$, and $\mathrm{dTHD} / \mathrm{dt}$ is obtained by comparing the output values of the components by calculated quantities as reference values. As a result, composite error of the algorithm is obtained, representing the accuracy of the algorithm. Decreasing the error value represents high sensitivity of the algorithm to measure and calculate the threshold values as criteria to determine ferroresonance of different types.

Besides, performance of digital components like comparators and logic gates to classify ferroresonance waveforms is crucial to sensitivity of the algorithm. In recent sensitivity analysis of the algorithm, a composite error of about $4 \%$ is obtained. Sensitivity of the digital components to classify ferroresonance modes is shown in Fig. 8. From the graph, harmonic ferroresonance is detected by the algorithm. According to criteria in Table 1, harmonic mode is detected when $\Delta \mathrm{f}$ holds a constant value above

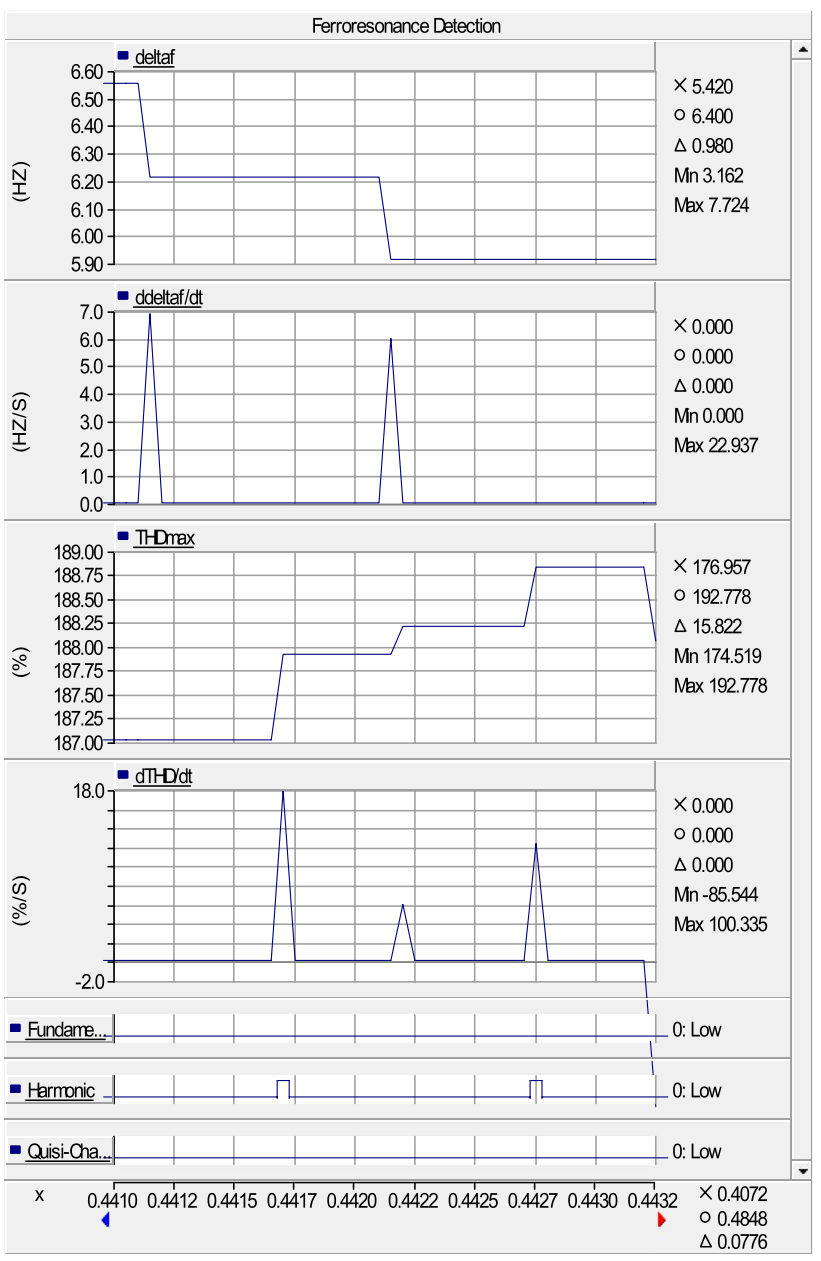

Fig. 8 Sensitivity of the algorithm to detect ferroresonance modes

zero, $\mathrm{d} \Delta \mathrm{f} / \mathrm{dt}$ becomes zero and the value of THD and $\mathrm{dTHD} / \mathrm{dt}$ keep the quantities more than $50 \%$ and $10 \% / \mathrm{S}$ respectively. As shown in the graph, derivative components follow exactly variation of $\Delta f$ and THD. In addition, comparators and logic gates detect harmonic ferroresonance mode according to specified threshold values. Hence, it is certified that by considering composite error of $4 \%$, logic components of the algorithm work correctly to classify ferroresonance of different types.

The setting quantities of NSDE are modified by protective algorithm, which is initiated by ferroresonance detection algorithm. As shown in Fig. 8, ferroresonance detection algorithm detects ferroresonance of different types discretely. It causes consecutive initiation and then outage of the relay setting modification algorithm. It brings about an inconsistency in setting quantities, in circumstances, where variety of ferroresonance modes is detected consecutively. It also drawbacks efficiency of the algorithm and causes decrement of reliability of protective system. 
To conquer such discordancy, a flip flap scheme can be utilized to be set based on detection of specified number of ferroresonance modes and then to be reset according to a specified quiescent period. The scheme requires a complementary study, which is out of scope of this work and can be considered as a future work.

\section{Structural Implementations}

In this section, structure of adaptive algorithm and protection strategy is implemented by PSCAD/EMTDC software for NSDE.

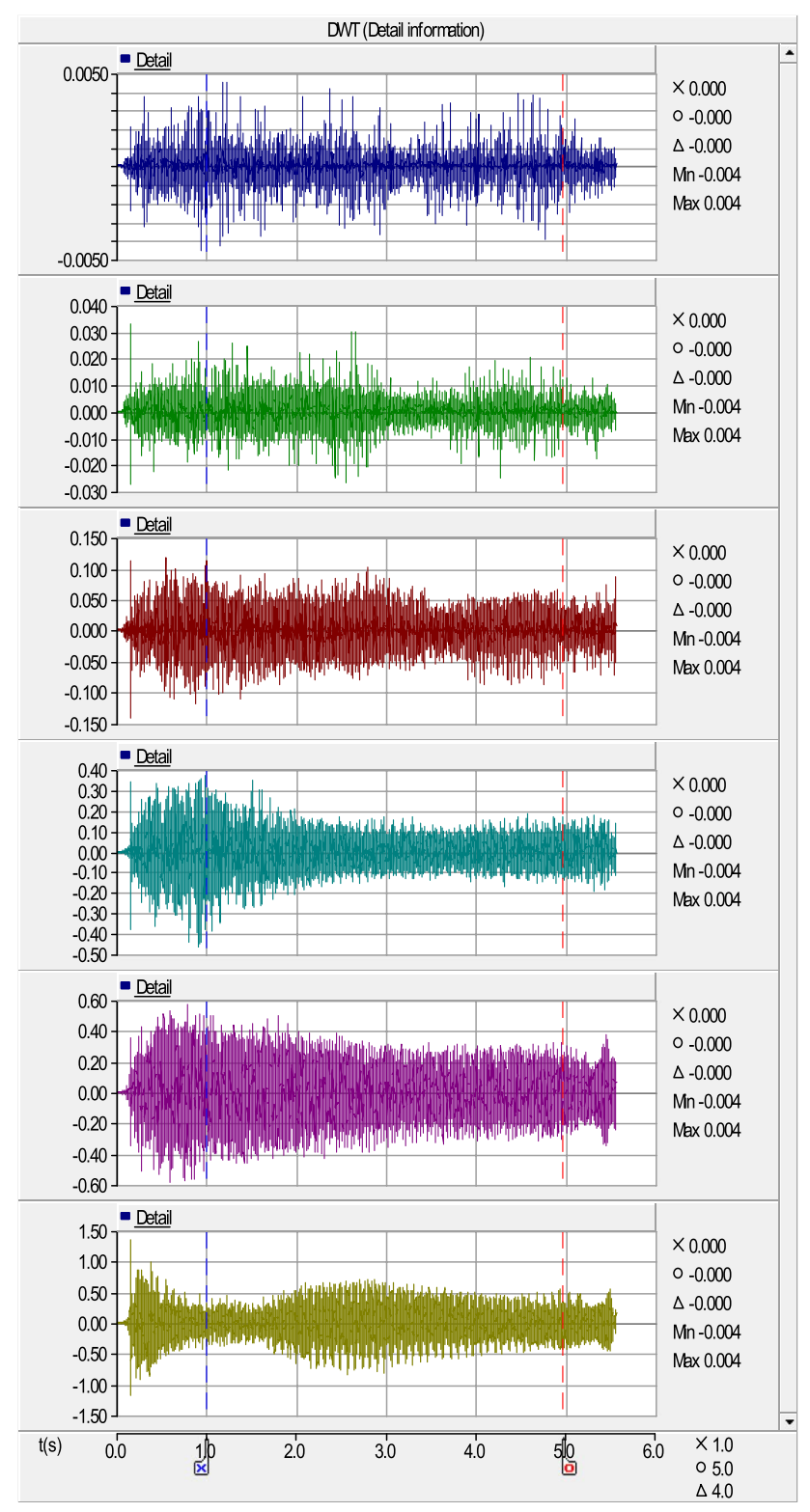

Fig. 9 Decomposition of ferroresonant signals

\section{Implementation of structure of the algorithm}

Logical and computational elements in the simulation software are employed to implement ferroresonance detection algorithm. Functionality of the algorithm is explained as follows.

Firstly, neural network is trained in commissioning term to determine several classes of nonlinearities and distinguishes ferroresonance waveforms from other nonlinear abnormalities. Variety of patterns respecting abnormalities is uploaded to the algorithm. Daubechies (db) mother wavelet of DWT decomposes the pattern signals up to six levels of approximation and detail information. Then, neural network is trained and clusters patterns into corresponding class. The algorithm is put into operation mode after termination of training process. As soon as recognition of ferroresonance criteria, neural network approves occurrence of ferroresonance according to pattern waveforms.

The algorithm also determines ferroresonance of different types by measuring THD and $\Delta \mathrm{f}$. The criteria in Table 1 are implemented by means of comparator and derivative tools and logic gates. In case of pickup operation of the relay or excessive true rms values of measured quantities along with determination of ferroresonance of different types, the corresponding signal is received by protection strategy. Then, protection strategy specifies the type of ferroresonance, in which relay setting modification must be implemented. The algorithm also receives the quantities of measured sequence components and previous relay setting values. Threshold values explained in Sect. 3.2 are generally specified by protection strategy logic. In addition to that, they can be determined by user in some special conditions.

The algorithm simulates the voltage increment versus variation of THD and $\Delta \mathrm{f}$ to determine stability domain boundary

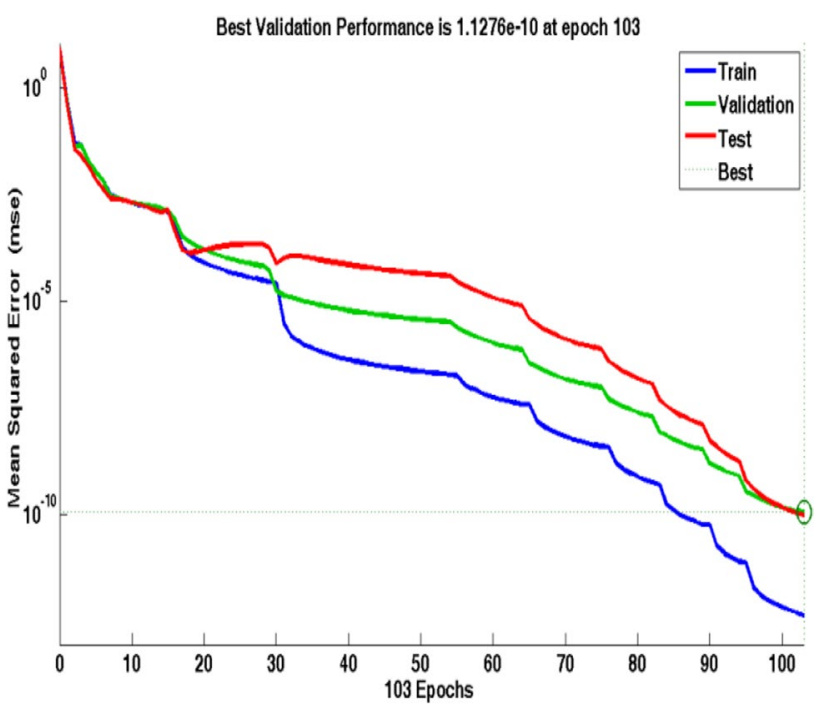

Fig. 10 Performance evaluation of neural network 
of voltage in the grid. To accomplish this, the "multiple run" property of the simulation software is utilized to ramp the magnitude of the voltage of all power sources to search for the values of voltages, which lead in occurrence of different modes of ferroresonance. Such characteristic of the algorithm is dramatically practical for protective system to forecast ferroresonance based on voltage variation in the network.

\section{Implementation of structure of the protection strategy}

As was mentioned in advance, protection strategy is responsible to determine behavior of the relay in effect of ferroresonance based on results of the studies, which are carried out by PSL. Here, structure of protection strategy is executed

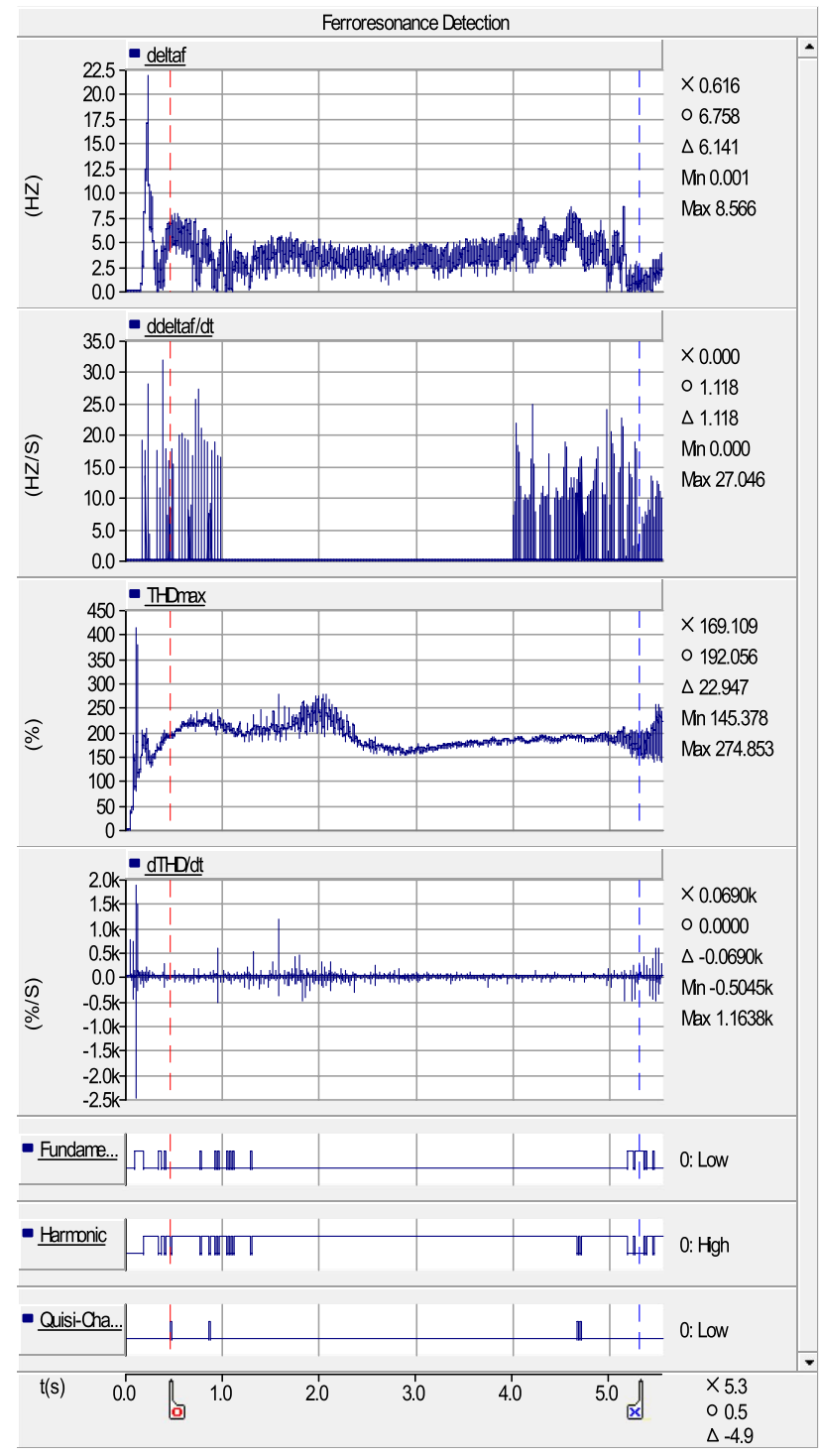

Fig. 11 Detection of ferroresonance of different types in the software to modify NSDE setting quantities as was described in detail in Sect. 3.3. Electronic and logical tools like, SR flip flap, comparator and logic gates are utilized to perform the protection strategy. As was discussed in advance, PSL determines threshold values and increasing coefficient values of PIF, SF, and TIF, in addition, decreasing coefficient values of PDF, SF, and TDF. However, in this study, the above-mentioned coefficients are considered constant to examine setting modification of the relay by the algorithm.

\section{Results and Discussion}

In this section, the results of designation and implementation of adaptive algorithm for NSDE is illustrated by examination of the algorithm in ferroresonance condition.

\section{Examination of the algorithm in the relay}

In this section, functionality of the adaptive algorithm designated for NSDE is examined in two different ferroresonant

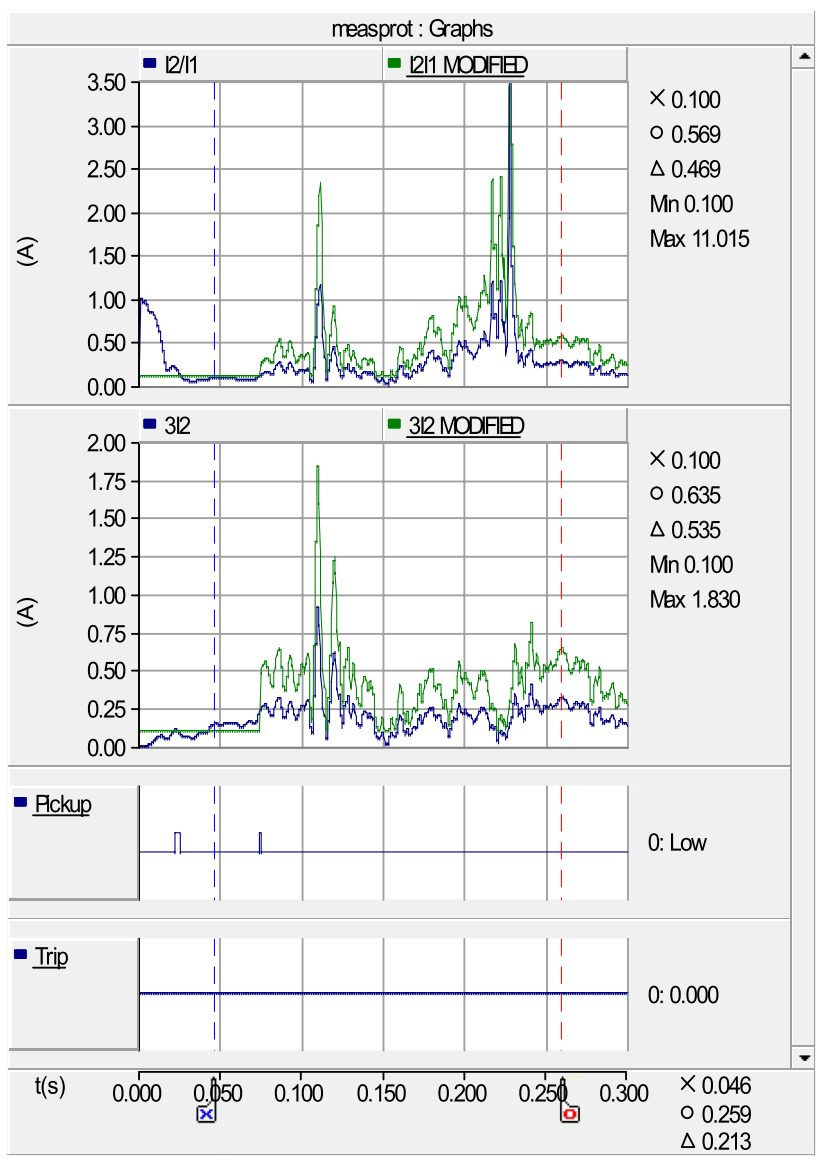

Fig. 12 Modification of NSDE setting quantities 
states to consider different aspects of operation of the algorithm.

First, pattern signals respecting ferroresonance and other nonlinearities are entered into the algorithm and then neural network is trained to distinguish different nonlinear abnormal conditions. Thereafter, DWT decomposes signals into six levels of detail information. Figure 9 shows decomposed ferroresonant signal, which is provided for training the neural network.

In order to evaluate performance of the neural network the algorithm utilizes Neural-fitting tool with Levenberg-Marquardt training algorithm and hidden Layer Size 10 in training validation and testing phases. As illustrated by Fig. 10, the best validation is $1.1276 \mathrm{e}-10$ obtained at epoch 103.

After that, the algorithm is put into operation to detect ferroresonant state, which was presented in Sect. 4. Current waveform is distinguished as ferroresonance by neural network then, variety of ferroresonance modes are recognized as shown in Fig. 11 by considering criteria listed in Table 1.

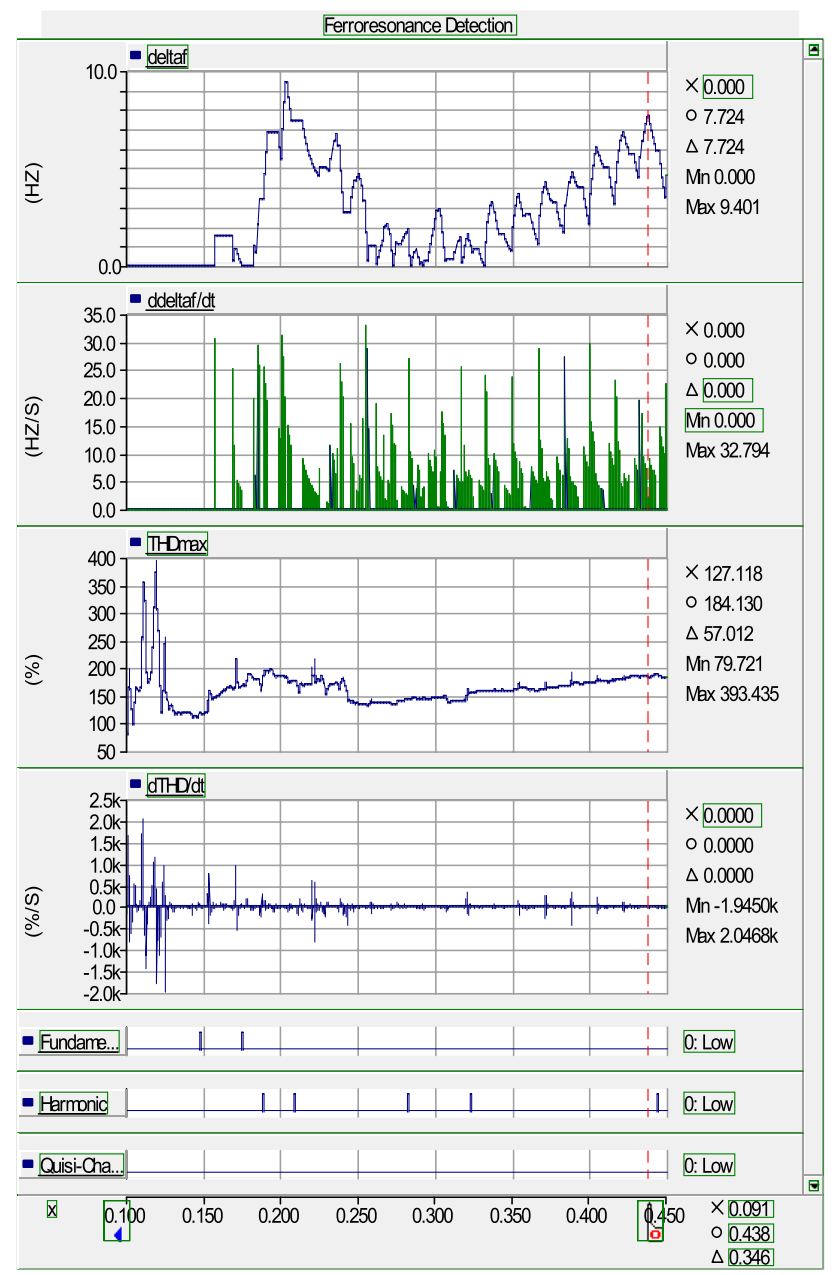

Fig. 13 Detection of ferroresonance of different types
First, fundamental ferroresonance is detected thereafter, the value of $\Delta \mathrm{f}$ rises up to $51 \mathrm{~Hz}$ as well as a significant rise in the values of $\mathrm{d} \Delta \mathrm{f} / \mathrm{dt}$, THD and $\mathrm{dTHD} / \mathrm{dt}$. Hence, harmonic, quasi-periodic and chaotic modes are also detected subsequently.

As was shown in Fig. 7, the ferroresonance leads in activation of pickup operation of the relay. Protection strategy determines activation of the algorithm by detecting fundamental ferroresonance type, which occurs at the time of 0.05 $\mathrm{S}$. At this time, the algorithm receives modification command to raise pickup quantities of the relay like; I2/I1 and I2forward. As illustrated in Fig. 12, setting quantities are raised by the PIF of PIF of about 2, which is specified by PSL. Consequently, it restrains mal operation of the relay in ferroresonance.

Changing configuration of the network like switching the lines in ferroresonance causes evolving different ferroresonant states. As shown in Fig. 13, ferroresonance detection algorithm detects ferroresonance of different types discretely. That is to say, there are quiescent periods, in which no ferroresonance is detected because the required criteria to detect ferroresonance of a specific mode cannot be provided at the same time. As was discussed in advance, it causes consecutive initiation and then outage of the relay setting modification algorithm. It brings about an inconsistency in setting quantities. In such situation, in order to illustrate

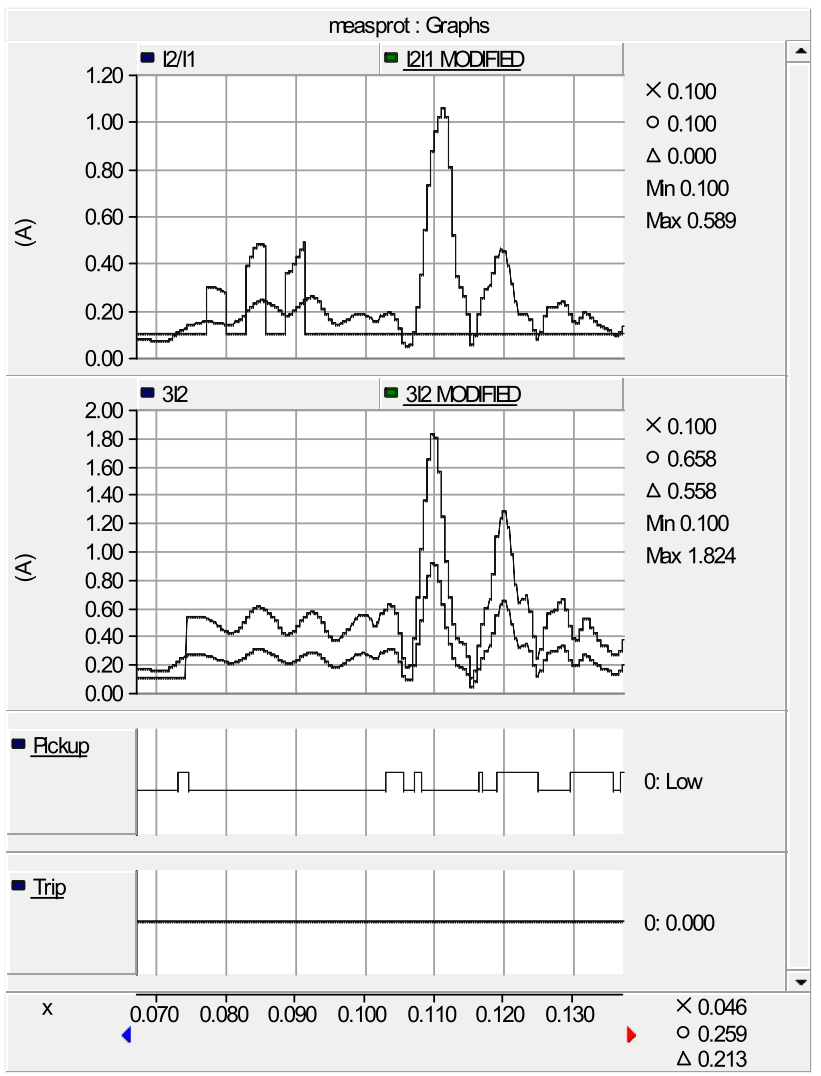

Fig. 14 Determination of stability domain boundary of voltage 
impact of discrete detection of ferroresonance modes on setting modification of NSDE, a rudimentary flip flap scheme, which was mentioned in Sect. 4.4, is utilized to modification of $3 \mathrm{I} 2$ parameter.

As shown in Fig. 14, the quantity of $3 \mathrm{I} 2$ is modified correctly, as the setting modification circuit is set by an RS flip flap as soon as the first ferroresonance mode is detected along with pickup operation of NSED. Whereas, the quantity of I2/I1 cannot be modified correctly as setting modification circuit is initiated and interrupted consecutively due to discrete detection of ferroresonance modes. As was discussed in Sect. 4.4, implementation of an efficient flip flap scheme requires a complementary study. Especially, reset characteristic of the flip flap scheme is one of the greatest challenges, which causes a comprehensive flip flap scheme is considered as a future work.

\section{Determination of stability domain boundary of voltage}

As was discussed in advance, the algorithm is responsible to specify stability domain boundary of voltage in the grid. This characteristic is beneficial for the system to predict probability of occurrence of variety of ferroresonance modes based on voltage increment versus THD and $\Delta \mathrm{f}$. The grid configuration introduced in Sect. 4 is experimented by this characteristic to examine variety of ferroresonant states experienced in voltage variations. As illustrated by Fig. 15, no-ferroresonance is detected in the area specified with horizontal green line. In case of increasing the voltage up to $140 \mathrm{kV}$, the value of THD increases while $\Delta \mathrm{f}$ is almost zero. This is the evidence of occurrence of fundamental ferroresonance. Then, non-fundamental ferroresonant states like; harmonic,

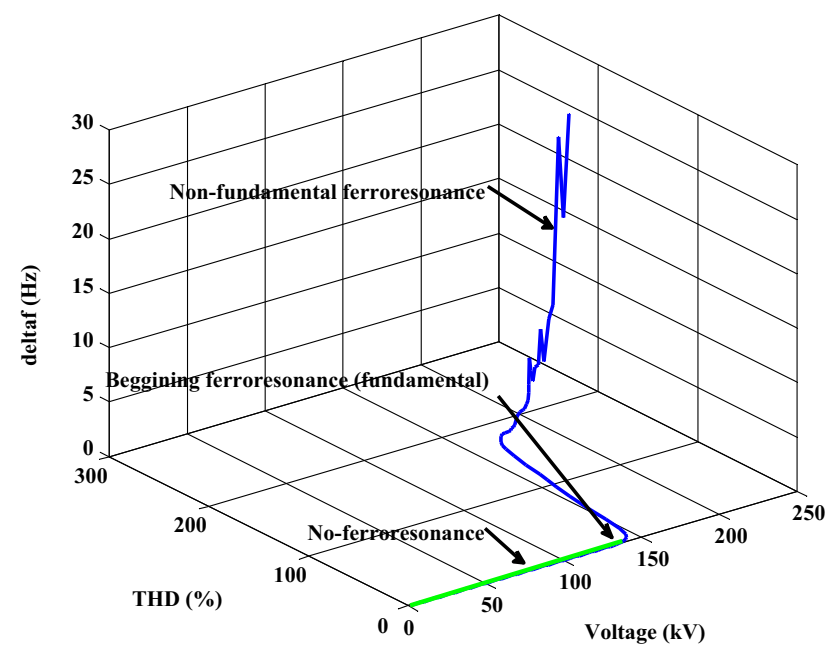

Fig. 15 Modification of NSDE setting quantities quasi-periodic and chaotic modes are recognized when the voltage increases up to $190 \mathrm{kV}$. In this time, the values of $\Delta \mathrm{f}$ and THD increase up to $25 \mathrm{~Hz}$ and $250 \%$ respectively.

\section{Conclusions}

It is proven that occurrence of ferroresonance causes mal operation of protective relays. In order to increase reliability of NSDE in smart grid adaptive algorithm based on time domain analysis of ferroresonance modifies setting quantities of the relay during ferroresonance. It firmly increases percentage of security and dependability of protection in smart grid. The algorithm is compatible with smart grid protection strategy and receives modification commands from the PSL based on results of electrical studies implemented in the network. The algorithm fairly discriminates ferroresonance from other nonlinear abnormalities in the network. In case of pickup operation of the relay or exponential increasing true rms value of negative sequence current in ferroresonance the algorithm decide to modify setting quantities of the relay to restrict operation or activates operation of the relay respectively. The algorithm has been experimented under variety of ferroresonant configurations to certify correctness of functionality. It is confirmed that the algorithm operates appropriately respecting reasonable modification of NSDE setting quantities to conform to protection strategy of smart grid.

Abbreviations DG: Distributed Generations; DFIG: Doubly-Fed Induction Generator; NSDE: Negative Sequence Directional Element; DWT: Discrete Wavelet Transform; $\Delta$ f: Frequency difference; THD: Total Harmonic Distortion; db: Daubechies mother wavelet; PSL: Protection Strategy Logic; OC: Over Current; PIF: Pickup Increasing Factor; SF: Security Factor; TIF: Time Increasing Factor; PDF: Pickup Decreasing Factor; TDF: Time Decreasing Factor; $\mathrm{A}_{\text {sec }}$ : Ampere in secondary side of instrument transformer

Acknowledgements The views and opinions of authors expressed in this paper do not necessarily state or reflect those of the (KPGM) Co. or anybody else.

Authors' contributions The corresponding author is single author of the manuscript who provides all materials ranging from research in development of the idea to writing and proofreading of the manuscript.

Availability of data and materials Reader may need to contact the corresponding author and get permission to have full access to the data.

\section{Declarations}

Competing interests The author declares that he has no competing interests. 


\section{References}

1. Momoh J (2012) SMART GRID fundamentals of design and analysis. IEEE Press, Published by John Wiley \& Sons, Inc., Hoboken, New Jersey, p. xiii

2. Aljahani ME (2014) An enhanced self-healing protection system in smart grid: using advanced and intelligent devices and applying hierarchical routing in sensor network technique. Master of Science dissertation Dep. Electrical and Computer Engineering Western Michigan Univ. June 2014

3. Esmaeili M, Rostami M, Gharehpetian GB, McInnis CP (2015) Ferroresonance after islanding of synchronous machine-based distributed generation. Can J Electr Comput Eng 38(2):154-161

4. Esmaeili M, Rostami M, Gharehpetian GB (2014) The effect of various types of distributed generations on ferroresonance, 22nd Iranian Conference on Electrical Engineering (ICEE), pp. $803-807$

5. Monadi M, Luna A, Candela JI, Rocabert J, Fayezizadeh M, Rodriguez P (2013) Analysis of ferroresonance effects in distribution networks with distributed source units. IECON, 39th Annual Conference of the IEEE Industrial Electronics Society, pp. $1974-1979$

6. Zheng TY, Kim YH, Kang YC (2011) Protection for a wind turbine generator in a large wind farm. Journal of Electrical Engineering \& Technology 6(4):466-473

7. Jones D, Bennett K (2012) Wind farm collector protection using directional overcurrent elements. IEEE PES Transmission and Distribution Conference and Exposition (T\&D), FL. USA, pp. $1-8$

8. Jing X, Zhong J, Lin S, Zhan C (2013) A novel overcurrent protection method based on wide area measurement in smart grid. IEEE Grenoble Conference, pp. $1-6$

9. Bujanovic T, Ghosh P (2016) Adaptive algorithm for microprocessor based distance relays in smart grid. 2016 the 4th IEEE International Conference on Smart Energy Grid Engineering, pp. $358-364$

10. Shih MY, Conde A, Leonowicz Z, Martirano L (2017) An adaptive overcurrent coordination scheme to improve relay sensitivity and overcome drawbacks due to distributed generation in smart grids. IEEE Transactions on Industry Applications

11. Mediha Mehmed-Hamza, Plamen Stanchev, "Overcurrent Protection Against Faults in Smart Grids",

12. Aktaibi A, George G, Rahman MA (2016) A high-speed digital current differential protection algorithm for power transmission lines in smart grids. IEEE Electrical Power and Energy Conference (EPEC)

13. Abdulwahid AH. A new concept of an intelligent protection system based on a discrete wavelet Transform and Neural Network Method for Smart Grids'

14. Maqbool U, Khan UA. Dynamic and transient analysis of a wavelet-based protection scheme for smart grids

15. Ocampo-Wilches JA, Ustariz-Farfan AJ, Cano-Plata EA. Modeling of a centralized microgrid protection scheme'

16. Liao J, Zhu X, Wang Q (2019) Transient current similaritybased protection for interconnecting transformers in wind farms. IEEE Access, vol. 7

17. Mohamed A, Sayed A-e, Moussa S, Elsamahy A (2019) Wind farm protection based on directional algorithm and positive sequence current with and without fiber optic backbone. 21St International Middle East Power Systems Conference (MEPCON)

18. Siahpoosh MK, Dorrell D, Li L. Ferroresonance assessment in a case study wind farm with 8 units of 2 MVA DFIG Wind Turbines"
19. George V, Kodeeswara Kumaran G, Shivashankari J, Dr. Ashok S (2016) Analysis of ferroresonance in a hybrid micro-grid with multiple distributed resources. International Conference on Electrical, Electronics, and Optimization Techniques (ICEEOT)

20. Rezaei S (2015) Impact of ferroresonance on protective relays in Manitoba Hydro $230 \mathrm{kV}$ electrical network", Proc. IEEE 15th Int. Conf. on Environment and Electrical Engineering Rome, Italy, pp. 1694 - 1699

21. Rezaei S (2018) Adaptive overcurrent protection against ferroresonance. IET Generation, Transmission \& Distribution, 12(7): $1573-1584$

22. Rezaei S (2019) An intelligent algorithm for negative sequence directional element of dfig during ferroresonance in smart grid. Proc. IEEE 19th Int. Conf. on Environment and Electrical Engineering and IEEE Industrial and Commercial Power Systems Europe (EEEIC / I\&CPS Europe), Italy

23. Roberts J, Guzmán A. Directional element design and evaluation," Schweitzer Engineering Laboratories, Inc. Pullman, WA USA

24. Elmore W, Price E (2000) Polarization Fundamentals. 27th Annual WPRC, Spokane, WA, Oct. 24-26, 2000

25. Elmore WA. Protective relaying theory and applications. 2nd edition, Revised and Expanded, Chapter 12, Section 3. 4, pp 243-244.

26. Yanjun L, Chunxia Z, Zhihua Z, Zichao J (2017) Fault identification method during oscillating based on negative sequence directional element. Int Conf on Power System Technology, China, pp 2537-2542

27. Karady GG, Mandava P (2014) Pilot directional protection using negative sequence directional element. PSC, Clemson uni., SC. USA. pp. 1-5

28. Manitoba HVDC Research Centre, "PSCAD X4 Online Help", Last Updated: 2012-06-12. Manitoba, Canada

29. Mokryani G, Haghifam MR (2008) Application of wavelet transform and MLP neural network for Ferroresonance identification. 2008 IEEE Power and Energy Society General Meeting - Conversion and Delivery of Electrical Energy in the 21st Century, pp. $1-6$

30. Goswami JC, Chan AK (1995) Fundamentals of wavelets: theory, algorithms, and applications, a wiley-interscience publication

31. Borges HB, Nievola JC (2012) Hierarchical classification using a Competitive Neural Network, $8^{\text {th }}$ International Conference on Natural Computation (ICNC), Chongqing, China, pp. 172-177

32. Jacobson DAN, Lehn PW, Menzies RW (2002) Stability domain calculations of period-1 ferroresonance in a nonlinear resonant circuit. IEEE Trans PWRD 17(3):865-871

33. Ph. Ferracci (1998) ferroresonance- Cahier technique", Schneider $n^{\circ} 190$, Groupe Schneider

34. Jacobson DAN (2003) Examples of ferroresonance in a high voltage power system'. Proc. IEEE PES General Meeting, pp. $1206-1212$

35. Jacobson DAN, Marti L (1999) Modeling ferroresonance in a 230 $\mathrm{kV}$ transformer-terminated double-circuit transmission line. Proc IPST Conf, Budapest- Hungary

36. Rezaei S (2016) Prevention of false operation of distance relay in ferroresonance. IJAREEIE 5(7): 5801-5814

37. Rezaei S (2017) Impact of plant outage on ferroresonance and mal operation of differential protection in presence of SVC in electrical network. IET Generation, Transmission \& Distribution 11(7): 1671-1682

38. Valverde V, Buigues G, Mazón AJ, Zamora I, Albizu I (2012) Ferroresonant configurations in power system', International Conference on Renewable Energies and Power Quality (ICREPQ'12), Santiago de Compostela (Spain), 28th to 30th March, 2012

39. Rezaei $S$ (2017) Impact of transmission line and plant outage on ferroresonance in AC transmission system and new suppression 
method', 13th IET International Conference on AC and DC Power Transmission (ACDC 2017) UK, pp.1-6

40. Rezaei S (2019) Adaptive algorithm in distance relay compatible with smart grid requirements during Sub Synchronous Resonance in transmission line', 19th IEEE International Conference on Environment and Electrical Engineering, 2019, \& 3th IEEE Industrial and Commercial Power Systems Europe (EEEIC / I\&CPS Europe), June 2019

41. S. Rezaei.: "Intelligent overcurrent protection during Ferroresonance in smart distribution grid", 19th IEEE International Conference on Environment and Electrical Engineering, 2019, \& 3th IEEE Industrial and Commercial Power Systems Europe (EEEIC / I\&CPS Europe), June 2019 .
42. Rezaei S (2018) Universal protection software and its application in smart grid. 18th IEEE International Conference on Environment and Electrical Engineering, 2018, \& 2th IEEE Industrial and Commercial Power Systems Europe (EEEIC / I\&CPS Europe), June 2018. pp. 1-6

Publisher's Note Springer Nature remains neutral with regard to jurisdictional claims in published maps and institutional affiliations. 